\title{
Heptamethine Cyanine Dyes in the Design of Photoactive Carbon Nanomaterials
}

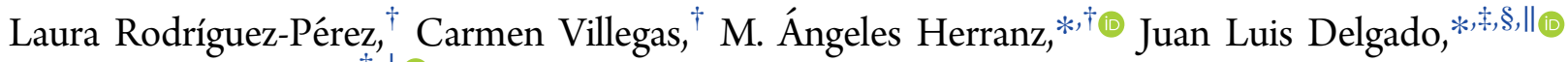 \\ and Nazario Martín* ${ }^{* \dagger, \perp},{ }^{\circ}$
}

\author{
${ }^{\dagger}$ Departamento de Química Orgánica, Facultad de Química, Universidad Complutense de Madrid, Avda. Complutense s/n, 28040 \\ Madrid, Spain \\ ${ }^{\ddagger}$ POLYMAT, University of the Basque Country UPV/EHU, Avenida de Tolosa 72, 20018 San Sebastian, Spain \\ ${ }^{\S}$ Faculty of Chemistry, University of the Basque Country UPV/EHU, P. Manuel Lardizabal 3, 20018 San Sebastian, Spain \\ "Ikerbasque, Basque Foundation for Science, Maria Diaz de Haro 3, 6 solairua, 48013 Bilbao, Spain \\ ${ }^{\perp}$ IMDEA-Nanociencia, c/Faraday 9, Ciudad Universitaria de Cantoblanco, 28049 Madrid, Spain
}

\section{Supporting Information}

ABSTRACT: Near-infrared (NIR) absorbing nanomaterials, built from anionic heptamethine cyanine dyes and single-walled carbon nanotubes or few-layer graphene, are presented. The covalent linkage, using 1,3-dipolar cycloaddition reactions, results in nanoconjugates that synchronize the properties of both materials, as demonstrated by an in-depth characterization study carried out by transmission electron microscopy, atomic force microscopy, thermogravimetric analysis, Fourier transform infrared spectroscopy, and X-ray photoelectron spectroscopy. UVvis-NIR and Raman spectroscopies further confirmed the unique electronic structure of the novel photoactive nanomaterials.

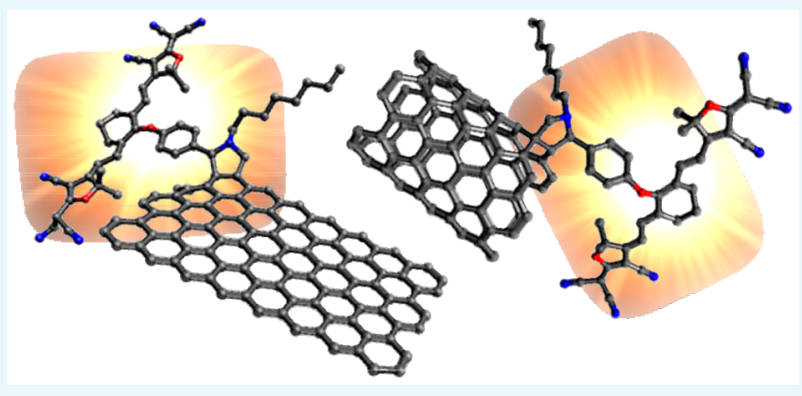

\section{INTRODUCTION}

The field of carbon nanostructures has attracted tremendous research interest over the last few years because of the fascinating properties and potential applications of these materials, which have stimulated scientists of different areas of knowledge. ${ }^{1}$ In particular, fullerenes, carbon nanotubes, and graphene have become extraordinarily popular nanomaterials because of their unique mechanical, electronic, and thermal properties. $^{2}$ However, it is often desirable to further modify their properties, and, in this sense, the use of a great variety of chemical methodologies has allowed the fine-tuning of carbon nanomaterial performance. ${ }^{3}$

The chemical modification of carbon nanotubes and graphene is critical to improve their processability for any practical application. Functionalization schemes that confer an additional element of control over the nanomaterial properties are particularly attractive, and, in this regard, the combination of carbon nanostructures with photo- and electroactive molecules or polymers attracts considerable attention for their potential as electron-donor/-acceptor systems for artificial photosynthesis, ${ }^{4}$ in photovoltaics, ${ }^{5}$ or as optical sensors. ${ }^{6}$

Interactions of carbon nanotubes and graphene with several photo- and electroactive molecules/macromolecules that operate in ultraviolet/visible regions of the spectrum, such as anthracene, ${ }^{7}$ pyrene, ${ }^{8}$ tetrathiafulvalenes, ${ }^{9}$ polymers, ${ }^{10}$ and other chromophores, ${ }^{11}$ have been studied. However, the nearinfrared (NIR) region, which constitutes a significant portion of the solar spectrum, is very promising for fluorescence detection and imaging and has been largely unexplored. In this regard, only a few examples combine carbon nanotubes and graphene with quantum dots ${ }^{12}$ and metalomacrocycles such as porphyrins and phthalocyanines. ${ }^{13}$ In particular, porphyrins are characterized by remarkably high extinction coefficients and tailor-made carbon nanohybrids endowed with porphyrins offer valuable new systems in the field of nano-optoelectronic devices for energy conversion, sensing, and biological applications. ${ }^{14}$

Anionic heptamethine cyanines represent another interesting class of ionic light harvester dyes showing an intense NIR absorption that spans from 750 to $900 \mathrm{~nm} .{ }^{15}$ Stimulated by the aforementioned results on porphyrinoid systems, we succeeded in preparing heptamethine cyanine molecular nanoconjugates 1-3 as NIR light harvesters in donor-acceptor systems (Figure 1). ${ }^{16-18}$ Here, we extend the methodology effectively used in the functionalization of $[60]$ and [70] fullerenes ${ }^{16}$ with heptamethine cyanines to single-walled carbon nanotubes (SWCNTs) and few-layer graphene (FLG) in an effort to increase the light-absorption capabilities of these carbon nanomaterials in the NIR region.

Received: October 6, 2017

Accepted: November 23, 2017

Published: December 22, 2017 

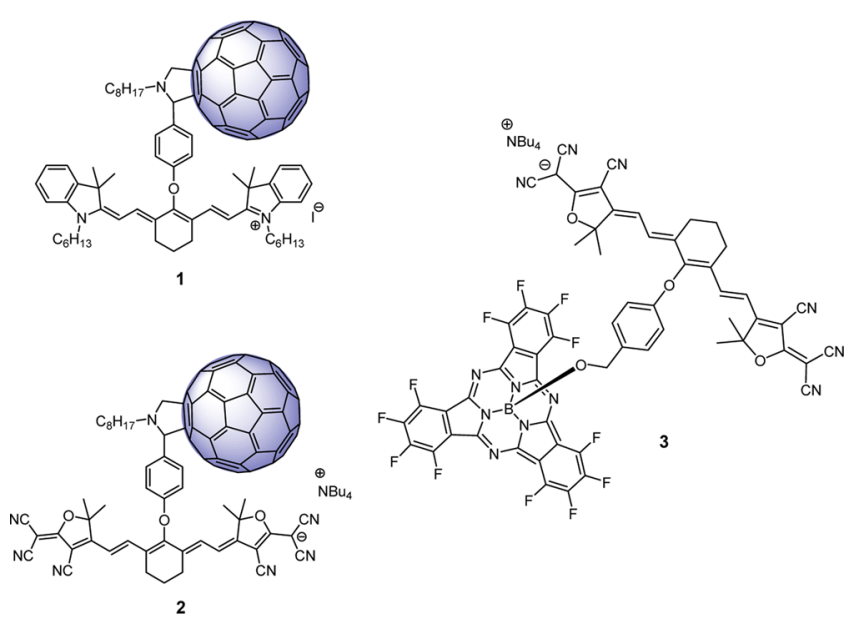

Figure 1. Cationic heptamethine cyanine-[60]fullerene $1,^{16 \mathrm{~b}}$ anionic heptamethine cyanine-[60]fullerene $2{ }^{16 a}$ and panchromatic light harvester $3 .{ }^{18}$

\section{RESULTS AND DISCUSSION}

Synthesis of Cyanine-Based Nanomaterials. The synthesis of the SWCNT-cyanine (5) and FLG-cyanine (6) nanoconjugates is described in Scheme 1. Formylcyanine 4 was

Scheme 1. Synthetic Approach Used for the Preparation of Carbon Nanomaterials 5 and 6

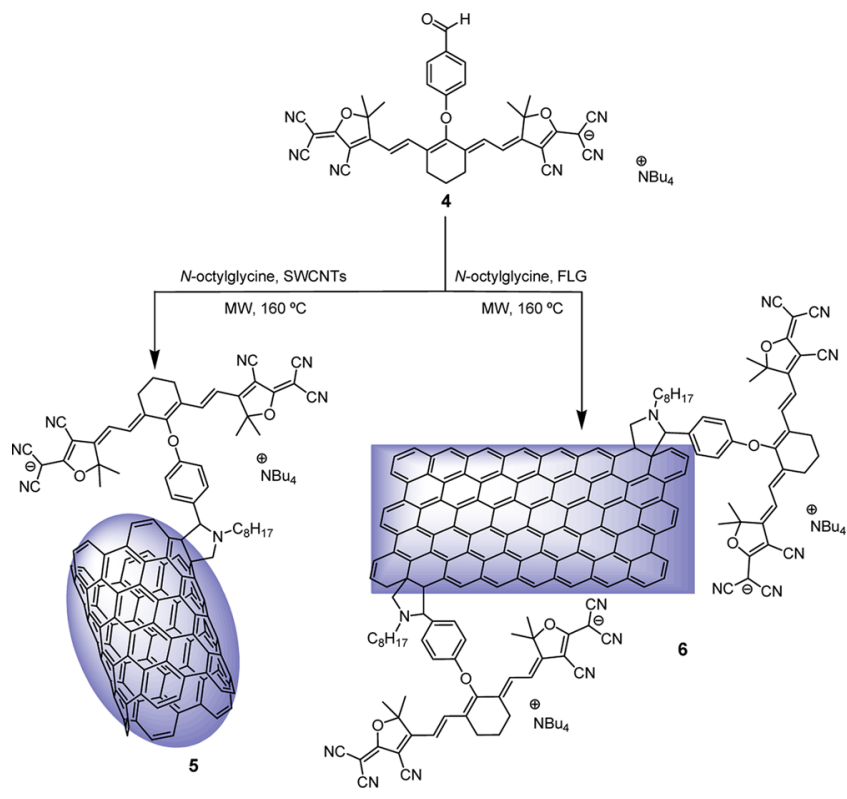

obtained following a previously reported procedure $^{16 a}$ and subsequently used in the 1,3-dipolar cycloaddition reaction ${ }^{19}$ with SWCNTs or FLG, and N-octylglycine-amino acid selected in an attempt to increase the solubility of these nanocarbons. The commercial SWCNTs were used without any treatment in the reaction, whereas FLG was obtained through graphite exfoliation in ortho-dichlorobenzene (oDCB) following Coleman's procedure. ${ }^{20}$ Such a FLG suspension in $o$ DCB was immediately reacted.

The 1,3-dipolar cycloaddition reaction was carried out under microwave irradiation in a closed quartz tube at $160{ }^{\circ} \mathrm{C}$. After cooling to room temperature, the solid was washed by filtration over a $0.2 \mu \mathrm{m}$ poly(tetrafluoroethylene) (PTFE) membrane several times with $o \mathrm{DCB}, \mathrm{CH}_{2} \mathrm{Cl}_{2}$, and $\mathrm{MeOH}$ (sonicated, centrifuged, and filtered) until the filtrate solution remained colorless, thus affording functionalized nanocarbons $\mathbf{5}$ and $\mathbf{6}$.

The obtained nanoconjugates were studied by a number of characterization techniques, including thermogravimetric analysis (TGA), Fourier transform infrared (FTIR) spectroscopy, Raman spectroscopy, X-ray photoelectron spectroscopy (XPS), atomic force microscopy (AFM), transmission electron microscopy (TEM), and UV-vis-NIR spectroscopy, to obtain fully detailed information about the structural, electronic, and chemical properties of the functionalized SWCNTs and FLG.

Structural Characterization. A first indication of the covalent attachment of the heptamethine cyanine to SWCNTs and FLG was obtained from TGA under an inert atmosphere (Figures 2 and S1, respectively). As anticipated, considering the

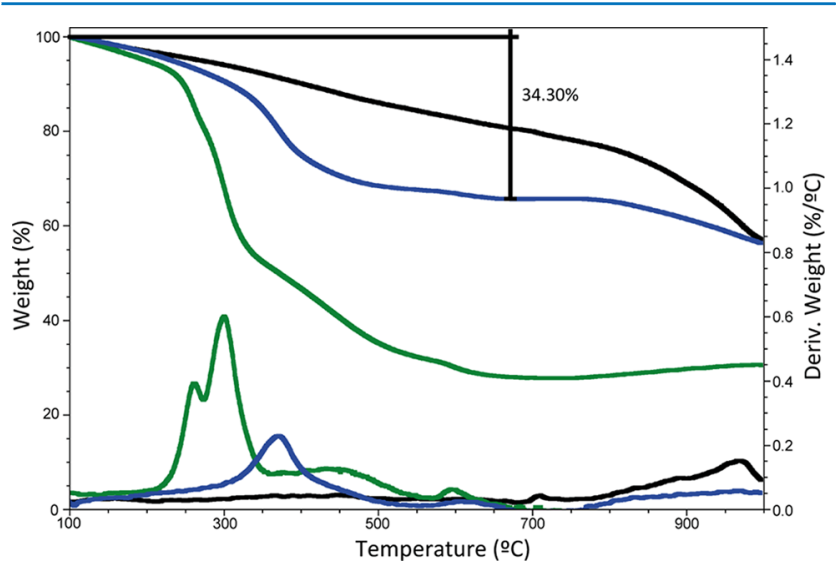

Figure 2. TGA analysis and first derivate curves of 5 (blue), 4 (green), and HiPco SWCNTs (black), recorded under nitrogen atmosphere.

different curvature and reactivity of the carbon nanostructures investigated, ${ }^{21}$ a slightly higher degree of functionalization was observed for SWCNTs (34\%) as compared to that for FLG (31\%) considering the mass loss of the materials at $650{ }^{\circ} \mathrm{C}$. From this data, we estimated 1 functional group per 140 carbon atoms in the case of nanoconjugate $\mathbf{5}$ and 1 functional group per 171 carbon atoms in the case of nanoconjugate 6. As expected, 5 and 6 maintain the decomposition pattern of the heptamethine cyanine, although with a small increase of the temperature decomposition maxima (see derivate curves in Figures 2 and S1), which indicates a thermal stabilization of the whole system due to the covalent linkage of heptamethine cyanine to SWCNTs or FLG.

Additional support of the 1,3-dipolar cycloaddition reaction taking place on SWCNTs and FLG was obtained by FTIR (Figures 3 and S2). The appearance of the stretching vibrations of the cyano groups at $2221 \mathrm{~cm}^{-1}$ for 5 and at $2218 \mathrm{~cm}^{-1}$ for 6 corroborates the existence of these groups on the surfaces of the nanoconjugates formed. Furthermore, the existence of other characteristic bands of heptamethine cyanine, such as those at ca. $1100-1096 \mathrm{~cm}^{-1}$, confirms the presence of heptamethine cyanine molecules in nanomaterials $\mathbf{5}$ and $\mathbf{6}$.

XPS provided a semiquantitative analysis of the elements found on the surface of $\mathbf{5}$ and $\mathbf{6}$, giving, in addition, information about the relative abundance of those elements within the material surface (Figures 4 and S3). ${ }^{22}$ Interestingly, in the XPS survey of nanocarbons 5 and 6 , in addition to the core-level contributions of $\mathrm{C} 1 \mathrm{~s}$ at $284.6 \mathrm{eV}$ and $\mathrm{O} 1 \mathrm{~s}$ at $532.6 \mathrm{eV}$, 


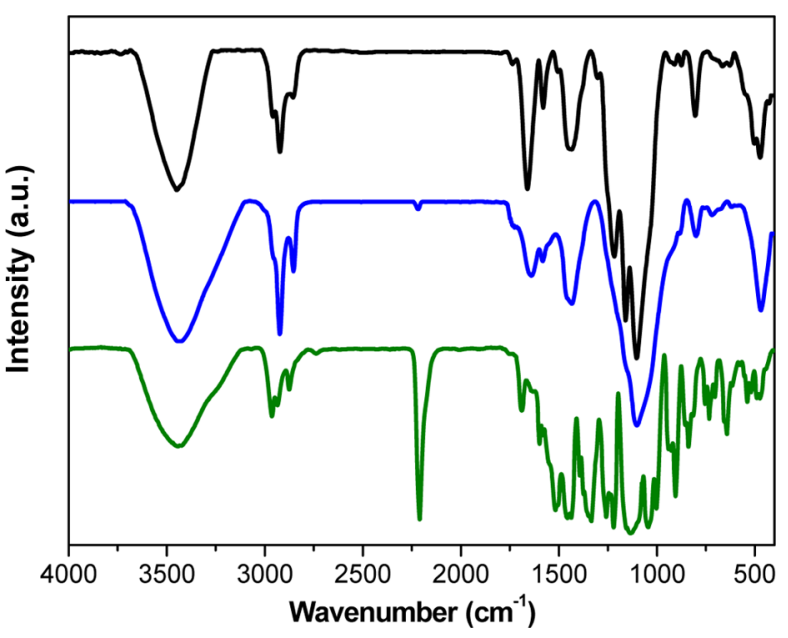

Figure 3. FTIR spectra of 6 (blue) relative to 4 (green) and FLG (black).

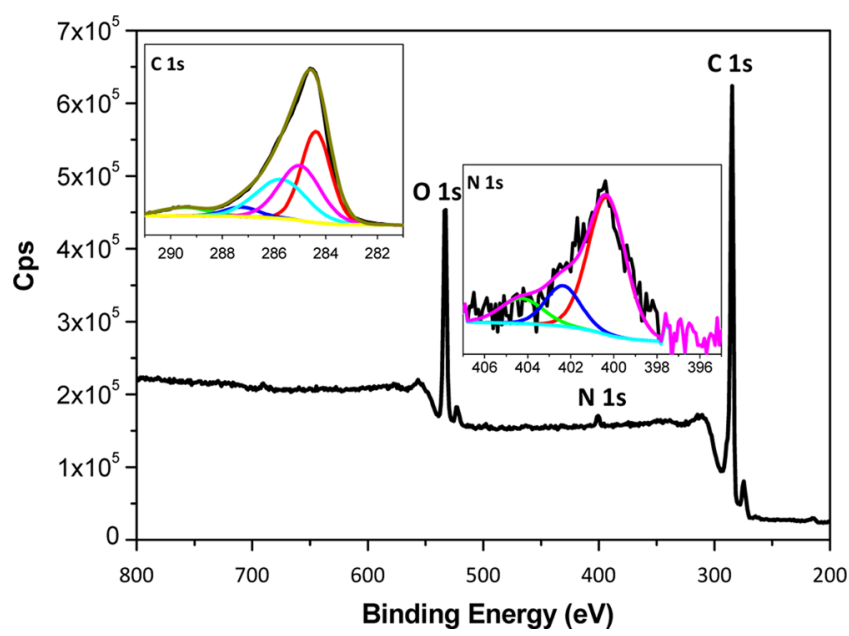

Figure 4. XPS analysis of FLG-cyanine nanoconjugates 6 . The inserts show the $\mathrm{C} 1 \mathrm{~s}$ and $\mathrm{N} 1 \mathrm{~s}$ core-level spectra deconvolution.

photoelectrons collected from the $\mathrm{N}$ 1s core level are also observed at $399.5 \mathrm{eV}$.

The XPS C 1s core-level spectrum of 6 can be deconvoluted into five peak components with binding energies of 284.4, 285.1, 285.8, 287.2, and $289.5 \mathrm{eV}$, which, similar to previous reports on covalently functionalized carbon nanomaterials, ${ }^{23}$ are assignable to $\mathrm{C}=\mathrm{C}, \mathrm{C}-\mathrm{C}, \mathrm{C}-\mathrm{N}, \mathrm{C}-\mathrm{O}$, and $\mathrm{C}=\mathrm{O}$ bonds, respectively (Figure 4 ). For $\mathbf{5}$, the same contributions to the $\mathrm{C}$ 1s core-level spectrum are found (Figure S3).

The analysis of the high-resolution $\mathrm{N}$ 1s core-level spectra of 5 and 6 furnished three different contributions. The first deconvoluted peak, observed at 404.3-403.5 eV, could be attributed to the quaternary ammonium salts, and the other two contributions found at lower binding energies could be attributed to the different types of cyano groups of the heptamethine cyanine core, which have slightly different binding energies, in the range from 399.5 to $402.4 \mathrm{eV}$, because they are differently involved in the stabilization of the negative charge density of the dye. ${ }^{24}$

Considered as a whole, XPS analysis of nanomaterials $\mathbf{5}$ and 6 demonstrates the presence of different nitrogenated functional groups (cyano, ammonium salts, and pyrrolidines) and,

ultimately, the covalent linkage of heptamethine cyanine to SWCNTs and FLG.

To complement the characterization of nanoconjugates 5 and 6, AFM and TEM investigations were employed to study their morphologies. ${ }^{25}$

From TEM, the disaggregation and dispersion of SWCNTs induced through covalent functionalization in nanoconjugates 5, when compared with a sample of commercial HiPco SWCNTs, are clearly observed (Figure S4). Furthermore, AFM reveals topographic images of commercial HiPco SWCNTs that present most of the SWCNTs stacked in aggregates of diameters up to $16 \mathrm{~nm}$, whereas in nanomaterial 5 , smaller aggregates, with diameters of 2-6 nm and typical lengths that range from $500 \mathrm{~nm}$ to $2 \mu \mathrm{m}$, are observed (Figure 5).
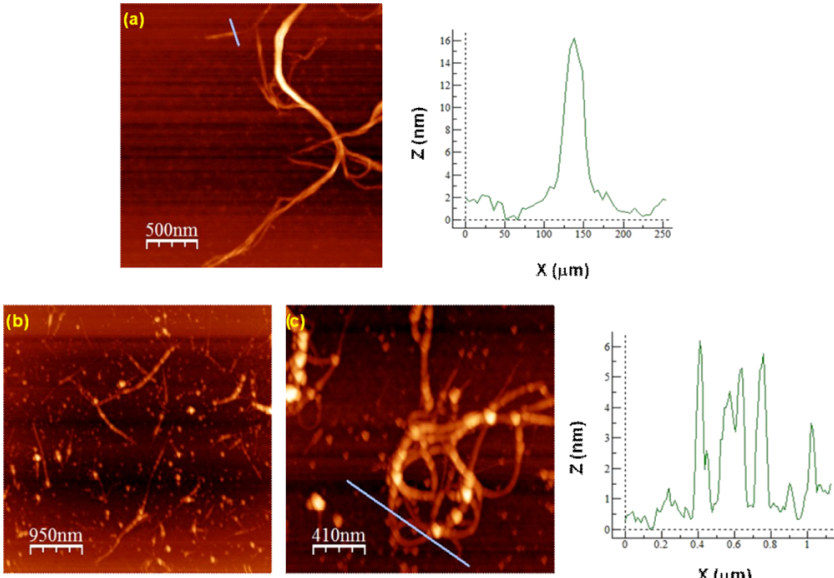

$x(\mu \mathrm{m})$

Figure 5. AFM images of: (a) commercial HiPco SWCNTs and (b, c) nanoconjugate 5. On the right, the height profile of each sample is displayed.

Similar to previous results, ${ }^{26}$ graphite exfoliation in $o$ DCB showed FLG nanosheets of less than five layers stacked with smaller flakes on their surface. The reaggregation of graphene flakes prior to functionalization was, to some extent, prevented by keeping the sample in solution because TEM analysis of nanoconjugate 6 reveals a nanomaterial that is uniformly disintegrated and with regular flakes that are randomly stacked onto each other (Figure 6).

Electronic Properties. Raman spectroscopy provides evidence of the covalent functionalization of SWCNTs and FLG at the time that allows to obtain valuable information about the structural and electronic characteristics of the nanomaterials. $^{27}$

The Raman spectrum of nanoconjugate $\mathbf{5}$ at $785 \mathrm{~nm}$ excitation is characteristic of a SWCNT-based nanomaterial, showing radial breathing modes (RBMs) between 170 and 310 $\mathrm{cm}^{-1}$, the $\mathrm{D}$ band, attributed to the disorder of the carbon hexagonal lattice on the SWCNT sidewalls ( $\mathrm{sp}^{3}$ carbons) at $1293 \mathrm{~cm}^{-1}$ and, the $\mathrm{G}$ mode or manual torque-tangential mode, which corresponds to the stretching mode in the graphite $\left(\mathrm{sp}^{2}\right.$ carbons), at $1593 \mathrm{~cm}^{-1}$ (Figure $7 \mathrm{a}$ ). On the basis of the comparison with the starting SWCNTs, several important conclusions are made: (i) RBM resonance signals reveal a pattern similar to that of the reference material and thus the reaction proceeds without preference for metallic or semiconducting tubes; ${ }^{28}$ (ii) the $\mathrm{D}$ band increases its intensity, $I_{\mathrm{D}} / I_{\mathrm{G}}$ $=0.1$ for 5 versus $I_{\mathrm{D}} / I_{\mathrm{G}}=0.06$ for the starting SWCNTs, which 

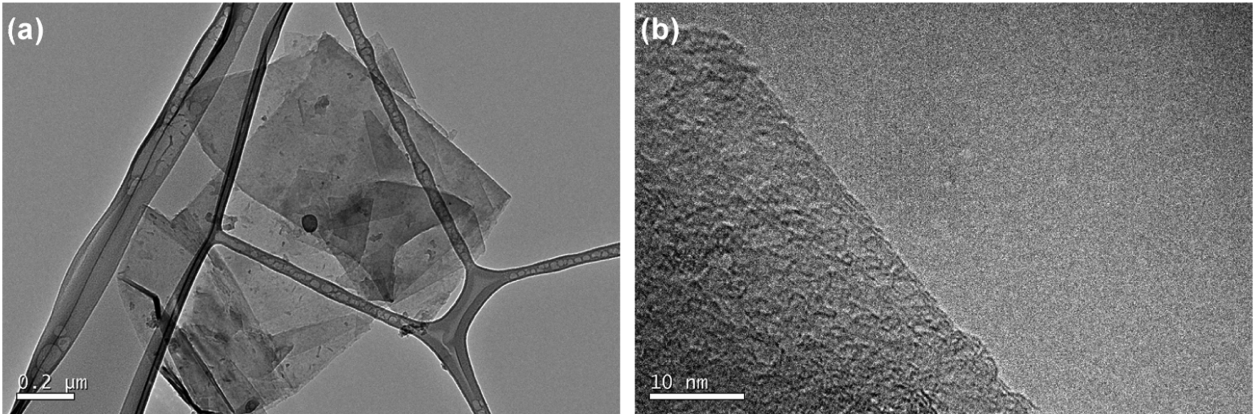

Figure 6. TEM images of nanoconjugate 6. (a) Scale bar $200 \mathrm{~nm}$ and (b) scale bar $10 \mathrm{~nm}$.
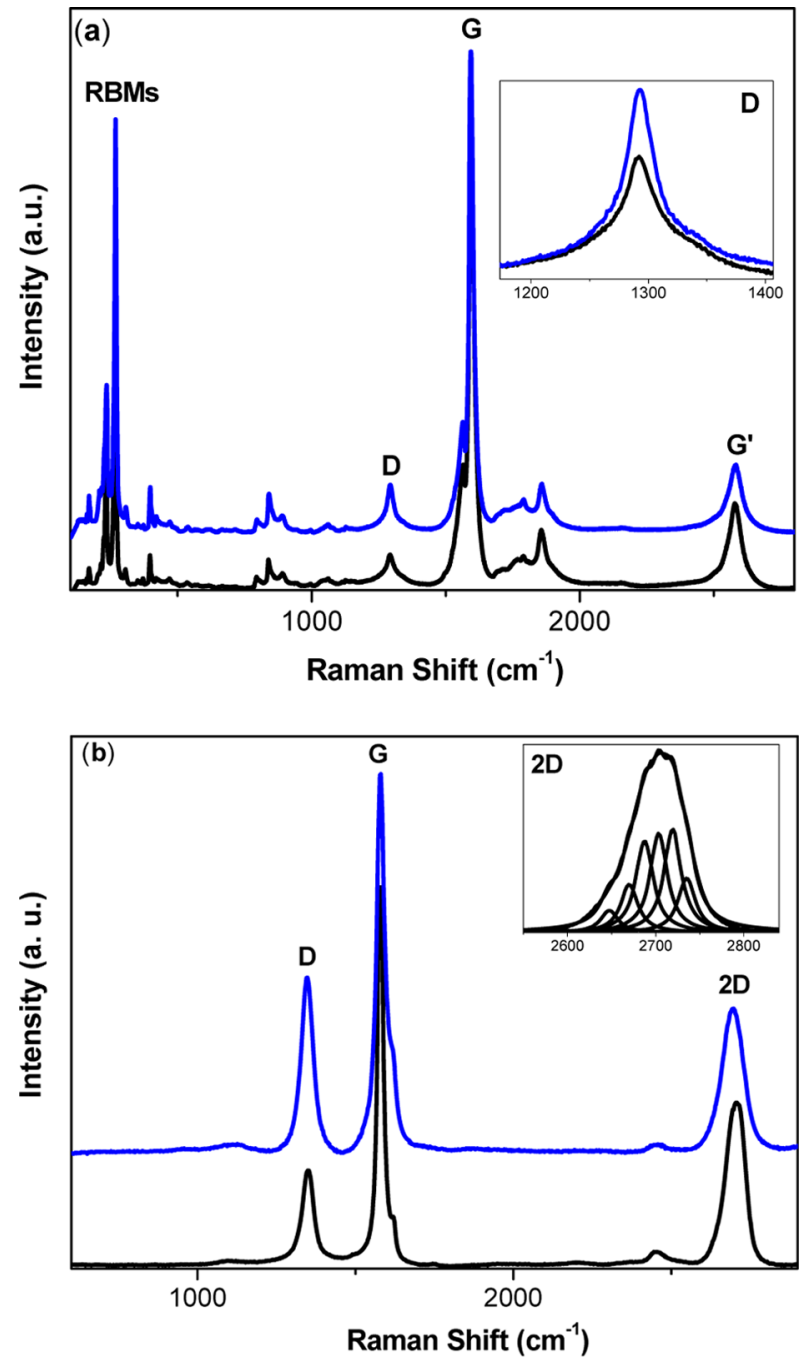

Figure 7. (a) Raman spectra of commercial HiPco SWCNTs (black) and 5 (blue) recorded under $785 \mathrm{~nm}$ excitation wavelength. The inset shows a comparison of D band intensities. (b) Raman spectra of 6 (blue) and exfoliated FLG (black) in $o$ DCB under $532 \mathrm{~nm}$ excitation wavelength. The inset shows the deconvolution of the twodimensional (2D) FLG band.

proves that the functionalization occurs through covalent bonding with the sidewall carbon atoms, which convert some $\mathrm{sp}^{2}$ bonding into $\mathrm{sp}^{3} ;{ }^{29}$ and (iii) no appreciable shifts are observed in the $\mathrm{G}$ or $\mathrm{G}^{\prime}$ modes of nanoconjugate 5 versus the starting SWCNTs and therefore a negligible electronic interaction seems to be operating in the ground state between heptamethine cyanine and SWCNTs. ${ }^{30}$

For nanoconjugate 6, the typical Raman bands of exfoliated graphite, namely, the $\mathrm{D}$ band at $1347 \mathrm{~cm}^{-1}$, the $\mathrm{G}$ band at 1578 $\mathrm{cm}^{-1}$, and the $2 \mathrm{D}$ band at $2694 \mathrm{~cm}^{-1}$, are observed by excitation at $532 \mathrm{~nm} .{ }^{31}$ Upon the formation of nanoconjugate 6 , the intensity of the $\mathrm{D}$ band increases compared to that for the exfoliated FLG because of the rehybridization of the carbon atoms from $\mathrm{sp}^{2}$ to $\mathrm{sp}^{3}$ (Figure $7 \mathrm{~b}$ ). Thus, the $I_{\mathrm{D}} / I_{\mathrm{G}}$ ratio increases from 0.25 for FLG to 0.46 for 6 , indicating the covalent anchoring of the heptamethine cyanine moiety to FLG. The 2D bands of both the exfoliated FLG and nanoconjugate 6 are highly symmetric and could be fitted by a single Lorentzian, which allows to determine the presence of FLG of around three sheets in the Raman study. ${ }^{32}$ Moreover, the $G$ band, which is very sensitive to doping effects, does not experience any shift from exfoliated FLG to nanoconjugate 6, which, similar to the Raman analysis of $\mathbf{5}$, indicates a weak or inexistent ground-state interaction between FLG and the dye.

Finally, the UV-vis-NIR analysis of $\mathbf{5}$ and $\mathbf{6}$, as well as that of formylcyanine 4, permits to corroborate the NIR absorption increase of FLG and SWCNTs with the covalent anchoring of heptamethine cyanine (Figures 8 and S5). All of the materials display the characteristic absorption peak of the dye at ca. 896 $\mathrm{nm}$. For nanoconjugate 5 , the absorption spectrum reflects the loss of the van Hove singularities of SWCNTs, an observation

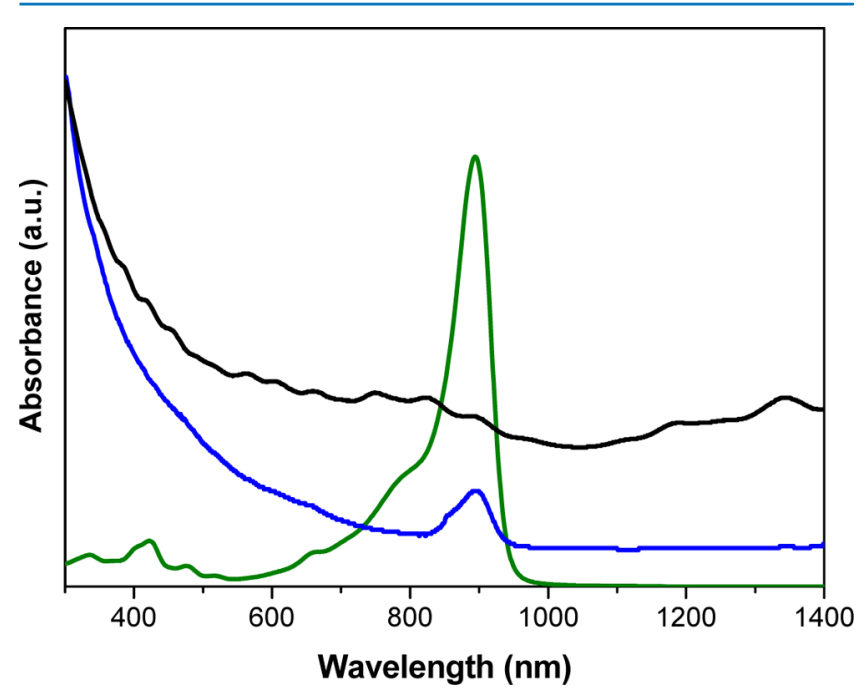

Figure 8. UV-vis-NIR spectra of 5 (blue) relative to 4 (green) and commercial HiPco SWCNTs (black) in $o$ DCB. 
that once again supports the covalent attachment of the heptamethine cyanine dye to the sidewalls of SWCNTs. ${ }^{33}$

\section{CONCLUSIONS}

In summary, we have carried out the synthesis of a new type of NIR absorbing nanomaterials, anionic heptamethine cyaninebased SWCNTs (5) and FLG (6) nanoconjugates, using 1,3dipolar cycloaddition reactions. The systematic and meticulous analyses through thermogravimetric analysis (TGA), FTIR spectroscopy, X-ray photoelectron spectroscopy (XPS), atomic force microscopy (AFM), and transmission electron microscopy (TEM) unambiguously confirmed the covalent attachment of the heptamethine cyanine moiety to the nanocarbons. Furthermore, the electronic characteristics of the nanoconjugates have been investigated considering Raman and UV-vis-NIR spectroscopies, which also confirmed the covalent linkage between the electroactive species. The new hybrid nanomaterials reveal appealing absorbance in the NIR region, thus paving the way to a variety of optoelectronic applications.

\section{EXPERIMENTAL SECTION}

Materials. HiPco SWCNTs were purchased from Carbon Nanotechnologies (lot: P0261, purity > 82, <18\% remaining iron particles, length $=100-1000 \mathrm{~nm}$, diameter $=0.8-1.4 \mathrm{~nm}$ ) and used without any further purification treatment. Graphite from TIMCAL (TIMREX SFG $15, \rho=2.26 \mathrm{~g} / \mathrm{cm}^{3}$, particle size $=8.80 \mu \mathrm{m}$, specific surface $=9.50 \mathrm{~m}^{2} / \mathrm{g}$, ashes $\leq 0.100 \%$, interlamellar distance $=3.354-3.358 \AA$ ) was used for the synthesis of few-layer graphene (FLG). Graphite flakes (200 $\mathrm{mg}$ ) were dispersed in anhydrous oDCB $(200 \mathrm{~mL})$ and sonicated in a low-power sonication bath for 150 min under an inert atmosphere to obtain FLG. The dispersion was centrifuged at $500 \mathrm{rpm}$ for $45 \mathrm{~min}$, and the supernatant was decanted and stored in solution.

Organic solvents and reagents used in this work were purchased from commercial suppliers and used as received, unless stated otherwise. Formylcyanine 4 (Scheme 1) was synthesized following the previously reported method. ${ }^{16 a}$

Instruments. TGA analyses were carried out under air and nitrogen in a TA-TGA-Q500 apparatus. The sample $(\sim 0.5 \mathrm{mg})$ was introduced inside a platinum crucible and equilibrated at $100{ }^{\circ} \mathrm{C}$, followed by a $10{ }^{\circ} \mathrm{C} \mathrm{min}^{-1}$ ramp between 100 and $1000{ }^{\circ} \mathrm{C}$ and by an isotherm of $30 \mathrm{~min}$. FTIR spectra were recorded in Bruker TENSOR 27 using a spectral range of $4000-400 \mathrm{~cm}^{-1}$, with a resolution of $1 \mathrm{~cm}^{-1}$, and in pellets of dispersed samples of the corresponding materials in dried $\mathrm{KBr}$. Raman spectra were recorded on Renishaw inVia (SWCNTs) or NT-MDT (FLG) microscopes at room temperature and at 785 or $532 \mathrm{~nm}$ wavelength, respectively. XPS analyses were carried out using a SPECS GmbH (PHOIBOS 150 9MCD) spectrometer operating in the constant analyzer energy mode. A nonmonochromatic aluminum X-ray source $(1486.61 \mathrm{eV})$ was used with a power of $200 \mathrm{~W}$ and voltage of $12 \mathrm{kV}$. Pass energies of 75 and $25 \mathrm{eV}$ were used for acquiring both survey and high-resolution spectra, respectively. Survey data were acquired from kinetic energies of $1487-400 \mathrm{eV}$ with an energy step of $1 \mathrm{eV}$ and $100 \mathrm{~ms}$ dwell time per point. The highresolution scans were taken around the emission lines of interest with $0.1 \mathrm{eV}$ steps and $100 \mathrm{~ms}$ dwell time per point. SpecsLab version 2.48 software was used for spectrometer control and data handling. The semiquantitative analysis was performed from the $\mathrm{C} 1 \mathrm{~s}(284.3 \mathrm{eV})$ signal. The samples were introduced as pellets of $8 \mathrm{~mm}$ diameter. UV-vis-NIR spectra were recorded in a UV-3600 Shimadzu spectrophotometer. TEM micrographs were obtained using a JEOL 2100 microscope operating at $200 \mathrm{kV}$. The samples were dispersed in $o \mathrm{DCB}$ and dropped onto a holey carbon copper grid (200 mesh), and the solvent was removed in a vacuum oven for $48 \mathrm{~h}$. AFM was performed on an SPM Nanoscope IIIa multimode microscope working in tapping mode with an RTESPSS tip (Veeco) at a working frequency of $\sim 235 \mathrm{kHz}$. The samples were prepared by spin-coating on mica.

Synthesis of SWCNT-Cyanine Nanoconjugate 5 . Formylcyanine $4(20 \mathrm{mg})$ and $N$-octylglycine $(20 \mathrm{mg})$ were added to pristine SWCNTs $(20 \mathrm{mg})$ suspended in $o$ DCB (10 $\mathrm{mL}$ ). The mixture was sonicated for $10 \mathrm{~min}$ and heated under microwave irradiation at $160{ }^{\circ} \mathrm{C}$ for $1 \mathrm{~h}$. The final product was separated from the reaction mixture by filtration over a poly(tetrafluoroethylene) (PTFE) membrane $(0.2 \mu \mathrm{m})$. The black solid was purified by successive washing in membrane with $o \mathrm{DCB}, \mathrm{CH}_{2} \mathrm{Cl}_{2}$, and $\mathrm{MeOH}$. FTIR (KBr): $\nu=2922,2854$, $2221,1718,1659,1601,1422$ (broad), 1260, 1096, 1021, and $800 \mathrm{~cm}^{-1}$. TGA: weight loss and temperature desorption (organic anchoring groups): $34.3 \%, 650{ }^{\circ} \mathrm{C}$. Raman: $I_{\mathrm{D}} / I_{\mathrm{G}}=$ 0.1. XPS: \% atomic: $\mathrm{C}(284.6 \mathrm{eV})=93.4, \mathrm{O}(532.6 \mathrm{eV})=5.5$, and $\mathrm{N}(399.5 \mathrm{eV})=1.1$. UV-vis-NIR $(o \mathrm{DCB}) \lambda_{\text {max }}: 896 \mathrm{~nm}$.

Synthesis of FLG-Cyanine Nanoconjugates 6. The exfoliated FLG in dry $o$ DCB was utilized as produced for further covalent modification. To a suspension of $25 \mathrm{~mL}$ of FLG in $o$ DCB were added formylcyanine $4(5 \mathrm{mg})$ and $N$ octylglycine $(5 \mathrm{mg})$. The reaction mixture was submitted for 12 microwave cycles following the sequence specified in Table 1.

Table 1. Irradiation Steps Used with the Microwave Reactor

\begin{tabular}{ccc} 
step & $T\left({ }^{\circ} \mathrm{C}\right)$ & hold time \\
1 & 130 & 1 \\
2 & 160 & 5 \\
3 & 50 & 1 \\
\hline
\end{tabular}

After cooling to room temperature, the resulting modified FLG was separated from the reaction mixture by filtration over a PTFE membrane $(0.2 \mu \mathrm{m})$. The black solid was purified by subsequent washing in membrane with oDCB, $\mathrm{CH}_{2} \mathrm{Cl}_{2}$, and MeOH. FTIR (KBr): $\nu=2924,2854,2218,1726,1641,1581$, $1460,1433,1157,1100$, and $800 \mathrm{~cm}^{-1}$. TGA: weight loss and temperature desorption (organic anchoring groups): 20.3\%, $400{ }^{\circ} \mathrm{C} ; 10.8 \%, 650{ }^{\circ} \mathrm{C}$. Raman: $I_{\mathrm{D}} / I_{\mathrm{G}}=0.45$. XPS: $\%$ atomic: $\mathrm{C}(284.6 \mathrm{eV})=81.0, \mathrm{O}(532.6 \mathrm{eV})=18.0$, and $\mathrm{N}(399.5 \mathrm{eV})=$ 1.0. UV-vis-NIR (oDCB) $\lambda_{\text {max }}: 896 \mathrm{~nm}$.

\section{ASSOCIATED CONTENT}

\section{Supporting Information}

The Supporting Information is available free of charge on the ACS Publications website at DOI: 10.1021/acsomega.7b01499.

Additional TGA and first derivative curves; FTIR spectra; XPS analysis; TEM images; and UV-vis-NIR spectra (PDF)

\section{AUTHOR INFORMATION}

\section{Corresponding Authors}

*E-mail: maherran@ucm.es (M.Á.H.).

*E-mail: juanluis.delgado@polymat.eu (J.L.D.). 
*E-mail: nazmar@ucm.es (N.M.).

\section{ORCID $\odot$}

M. Ángeles Herranz: 0000-0001-9155-134X

Juan Luis Delgado: 0000-0002-6948-8062

Nazario Martín: 0000-0002-5355-1477

\section{Notes}

The authors declare no competing financial interest.

\section{ACKNOWLEDGMENTS}

Financial support from the European Research Council (ERC320441-Chiralcarbon), the Ministerio de Economía y Competitividad (MINECO) of Spain (Projects CTQ2014-52045-R, CTQ2015-71936-REDT, and CTQ2015-70921-P), and the CAM (PHOTOCARBON project S2013/MIT-2841) is acknowledged. J.L.D. thanks Ikerbasque, the Basque Foundation for Science, for an "Ikerbasque Research Fellow" contract, Polymat Foundation, MINECO of Spain, for IEDI-2015-00666 grant and Iberdrola Foundation for financial support.

\section{REFERENCES}

(1) (a) Graphene: Synthesis, Properties, and Phenomena, 1st ed.; Rao, C. N. R., Sood, A. K., Eds.; Wiley-VCH Verlag \& Co.: Weinheim, 2013. (b) Fullerenes Principles and Applications, 2nd ed.; Langa, F., Nierengarten, J. F., Eds.; RSC: Cambridge, 2012. (c) Carbon Nanotubes and Related Structures, 1st ed.; Guldi, D. M., Martín, N., Eds.; Wiley-VCH Verlag \& Co.: Weinheim, 2010. (d) Delgado, J. L.; Herranz, M. A.; Martín, N. The nano-forms of carbon. J. Mater. Chem. 2008, 18, 1417-1426.

(2) (a) Chemical Synthesis and Applications of Graphene and Carbon Materials, 1st ed.; Antonietti, M., Mullen, K., Eds.; Wiley-VCH Verlag \& Co.: Weinheim, 2016. (b) Georgakilas, V.; Perman, J. A.; Tucek, J.; Zboril, R. Broad Family of Carbon Nanoallotropes: Classification, Chemistry, and Applications of Fullerenes, Carbon Dots, Nanotubes, Graphene, Nanodiamonds, and Combined Superstructures. Chem. Rev. 2015, 115, 4744-4822.

(3) (a) Miners, S. A.; Rance, G. A.; Khlobystov, A. N. Chemical reactions confined within carbon nanotubes. Chem. Soc. Rev. 2016, 45, 4727-4746. (b) Wang, X.; Shi, G. An introduction to the chemistry of graphene. Phys. Chem. Chem. Phys. 2015, 17, 28484-28504. (c) Vázquez, E.; Giacalone, F.; Prato, M. Non-conventional methods and media for the activation and manipulation of carbon nanoforms. Chem. Soc. Rev. 2014, 43, 58-69. (d) Eigler, S.; Hirsch, A. Chemistry with graphene and graphene oxide-challenges for synthetic chemists. Angew. Chem., Int. Ed. 2014, 53, 7720-7738. (e) Delgado, J. L.; Filippone, S.; Giacalone, F.; Herranz, M. A.; Illescas, B.; Pérez, E. M.; Martín, N. Buckyballs. Top. Curr. Chem. 2014, 350, 1-64. (f) Rodríguez-Pérez, L.; Herranz, M. A.; Martín, N. The chemistry of pristine graphene. Chem. Commun. 2013, 49, 3721-3735.

(4) (a) Bottari, G.; Herranz, M. A.; Wibmer, L.; Volland, M.; Rodríguez-Pérez, L.; Guldi, D. M.; Hirsch, A.; Martín, N.; D’Souza, F.; Torres, T. Chemical functionalization and characterization of graphene-based materials. Chem. Soc. Rev. 2017, 46, 4464-4500. (b) Dirian, K.; Herranz, M. A.; Katsukis, G.; Malig, J.; RodríguezPérez, L.; Romero-Nieto, C.; Strauss, V.; Martín, N.; Guldi, D. M. Low dimensional nanocarbons-chemistry and energy/electron transfer reactions. Chem. Sci. 2013, 4, 4335-4353. (c) D'Souza, F.; Ito, O. Photosensitized electron transfer processes of nanocarbons applicable to solar cells. Chem. Soc. Rev. 2012, 41, 86-96.

(5) (a) Martín, N. Carbon Nanoforms for Photovoltaics: Myth or Reality? Adv. Energy Mater. 2017, 7, No. 1601102. (b) Kucharski, T. J.; Ferralis, N.; Kolpak, A. M.; Zheng, J. O.; Nocera, D. G.; Grossman, J. C. Templated assembly of photoswitches significantly increases the energy-storage capacity of solar thermal fuels. Nat. Chem. 2014, 6, 441-447. (c) Xue, C.; Gao, M.; Xue, Y.; Zhu, L.; Dai, L.; Urbas, A.; Li, Q. Building 3D Layer-by-Layer Graphene-Gold Nanoparticle Hybrid Architecture with Tunable Interlayer Distance. J. Phys. Chem. C 2014,
118, 15332-15338. (d) Wang, H.-X.; Wang, Q.; Zhou, K.-G.; Zhang, H.-L. Graphene in Light: Design, Synthesis and Applications of Photoactive Graphene and Graphene-Like Materials. Small 2013, 9, 12661283.

(6) (a) Wang, L.; Bisoyi, H. K.; Zheng, Z.; Gutierrez-Cuevas, K. G.; Singh, G.; Kumar, S.; Bunning, T. J.; Li, Q. Stimuli-directed selforganized chiral superstructures for adaptive windows enabled by mesogen-functionalized graphene. Mater. Today 2017, 20, 230-237. (b) Heller, D. A.; Baik, S.; Eurell, T. E.; Strano, M. S. Single-Walled Carbon Nanotube Spectroscopy in Live Cells: Towards Long-Term Labels and Optical Sensors. Adv. Mater. 2005, 17, 2793-2799. (c) Loh, K. P.; Bao, Q.; Eda, G.; Chhowalla, M. Graphene oxide as a chemically tunable platform for optical applications. Nat. Chem. 2010, 2, 1015-1024.

(7) Zhang, J.; Lee, J.-K.; Wu, Y.; Murray, R. W. Photoluminescence and Electronic Interaction of Anthracene Derivatives Adsorbed on Sidewalls of Single-Walled Carbon Nanotubes. Nano Lett. 2003, 3, 403-407.

(8) Chen, R. J.; Zhang, Y.; Wang, D.; Dai, H. Noncovalent Sidewall Functionalization of Single-Walled Carbon Nanotubes for Protein Immobilization. J. Am. Chem. Soc. 2001, 123, 3838-3839.

(9) (a) López-Andarias, J.; López, J. L.; Atienza, C.; Brunetti, F. G.; Romero-Nieto, C.; Guldi, D. M.; Martín, N. Controlling the crystalline three-dimensional order in bulk materials by single-wall carbon nanotubes. Nat. Commun. 2014, 5, No. 3763. (b) Mateos-Gil, J.; Rodríguez-Pérez, L.; Moreno Oliva, M.; Katsukis, G.; Romero-Nieto, C.; Herranz, M. A.; Guldi, D. M.; Martín, N. Electroactive carbon nanoforms: a comparative study via sequential arylation and click chemistry reactions. Nanoscale 2015, 7, 1193-1200.

(10) Samanta, S. K.; Fritsch, M.; Scherf, U.; Gomulya, W.; Bisri, S. Z.; Loi, M. A. Conjugated polymer-assisted dispersion of single-wall carbon nanotubes: the power of polymer wrapping. Acc. Chem. Res. 2014, 47, 2446-2456.

(11) (a) Ozawa, H.; Kosaka, K.; Kita, T.; Yoshikawa, K.; Haga, M. Controlling the Direction of the Molecular Axis of Rod-Shaped Binuclear Ruthenium Complexes on Single-Walled Carbon Nanotubes. Chem. - Eur. J. 2016, 22, 6575-6582. (b) Setaro, A.; Bluemmel, P.; Maity, C.; Hecht, S.; Reich, S. Non-Covalent Functionalization of Individual Nanotubes with Spiropyran-Based Molecular Switches. Adv. Funct. Mater. 2012, 22, 2425-2431.

(12) Iacono, F.; Palencia, C.; de la Cueva, L.; Meyns, M.; Terracciano, L.; Vollmer, A.; de la Mata, M. J.; Klinke, C.; Gallego, J. M.; Juárez, B. H.; Otero, R. Interfacing Quantum Dots and Graphitic Surfaces with Chlorine Atomic Ligands. ACS Nano 2013, 7, 25592565.

(13) Bottari, G.; de la Torre, G.; Guldi, D. M.; Torres, T. Covalent and Noncovalent Phthalocyanine-Carbon Nanostructure Systems: Synthesis, Photoinduced Electron Transfer, and Application to Molecular Photovoltaics. Chem. Rev. 2010, 110, 6768-6816.

(14) (a) D'Souza, F.; Ito, O. Supramolecular donor-acceptor hybrids of porphyrins/phthalocyanines with fullerenes/carbon nanotubes: electron transfer, sensing, switching, and catalytic applications. Chem. Commun. 2009, 4913-4928. (b) Mao, B.; Calatayud, D. G.; Mirabello, V.; Kuganathan, N.; Ge, H.; Jacobs, R. M. J.; Shepherd, A. M.; Ribeiro Martins, J. A.; De La Serna, J. B.; Hodges, B. J.; Botchway, S. W.; Pascu, S. I. Fluorescence-Lifetime Imaging and Super-Resolution Microscopies Shed Light on the Directed- and Self-Assembly of Functional Porphyrins onto Carbon Nanotubes and Flat Surfaces. Chem. - Eur. J. 2017, 23, 9772-9789.

(15) Roth, A.; Schierl, C.; Ferrer-Ruiz, A.; Minameyer, M.; Rodríguez-Pérez, L.; Villegas, C.; Herranz, M. A.; Martín, N.; Guldi, D. M. Low-Dimensional Carbon Allotropes: Ground- and ExcitedState Charge Transfer with NIR-Absorbing Heptamethine Cyanine. Chem 2017, 3, 164-173.

(16) (a) Villegas, C.; Krokos, E.; Bouit, P.-A.; Delgado, J. L.; Guldi, D. M.; Martin, N. Efficient light harvesting anionic heptamethine cyanine-[60] and [70]fullerene hybrids. Energy Environ. Sci. 2011, 4, 679-684. (b) Bouit, P.-A.; Spänig, F.; Kuzmanich, G.; Krokos, E.; Oelsner, C.; Garcia-Garibay, M. A.; Delgado, J. L.; Martin, N.; Guldi, 
D. M. Efficient Utilization of Higher-Lying Excited States to Trigger Charge-Transfer Events. Chem. - Eur. J. 2010, 16, 9638-9645.

(17) Bouit, P.-A.; Rauh, D.; Neugebauer, S.; Delgado, J. L.; Di Piazza, E.; Rigaut, S.; Maury, O.; Andraud, C.; Dyakonov, V.; Martín, N. A "Cyanine-Cyanine" Salt Exhibiting Photovoltaic Properties. Org. Lett. 2009, 11, 4806-4809.

(18) Nieto, C. R.; Guilleme, J.; Villegas, C.; Delgado, J. L.; GonzálezRodríguez, D.; Martin, N.; Torres, T.; Guldi, D. M. Subphthalocyanine-polymethine cyanine conjugate: an all organic panchromatic light harvester that reveals charge transfer. J. Mater. Chem. 2011, 21, 15914-15918.

(19) (a) Tasis, D.; Tagmatarchis, N.; Bianco, A.; Prato, M. Chemistry of Carbon Nanotubes. Chem. Rev. 2006, 106, 1105-1136. (b) Maggini, M.; Scorrano, G.; Prato, M. Addition of azomethine ylides to $\mathrm{C}_{60}$ : synthesis, characterization, and functionalization of fullerene pyrrolidines. J. Am. Chem. Soc. 1993, 115, 9798-9799.

(20) (a) Coleman, J. N. Liquid Exfoliation of Defect-Free Graphene. Acc. Chem. Res. 2013, 46, 14-22. (b) Hernandez, Y.; Nicolosi, V.; Lotya, M.; Blighe, F. M.; Sun, Z.; De, S.; McGovern, I. T.; Holland, B.; Byrne, M.; Gun'Ko, Y. K.; Boland, J. J.; Niraj, P.; Duesberg, G.; Krishnamurthy, S.; Goodhue, R.; Hutchison, J.; Scardaci, V.; Ferrari, A. C.; Coleman, J. N. High-yield production of graphene by liquid-phase exfoliation of graphite. Nat. Nanotechnol. 2008, 3, 563-568.

(21) Hirsch, A.; Vostrowsky, O. Functionalization of Carbon Nanotubes. Top. Curr. Chem. 2005, 245, 193-237.

(22) Lipińska, M. E.; Rebelo, S. L. H.; Pereira, M. F. R.; Gomes, J. A. N. F.; Freire, C.; Figueiredo, J. L. New insights into the functionalization of multi-walled carbon nanotubes with aniline derivatives. Carbon 2012, 50, 3280-3294.

(23) (a) Okpalugo, T. I. T.; Papakonstantinou, P.; Murphy, H.; McLaughlin, J.; Brown, N. M. D. High resolution XPS characterization of chemical functionalised MWCNTs and SWCNTs. Carbon 2005, 43, 153-161. (b) Del Canto, E.; Flavin, K.; Movia, D.; Navio, C.; Bittencourt, C.; Giordani, S. Critical Investigation of Defect Site Functionalization on Single-Walled Carbon Nanotubes. Chem. Mater. 2011, 23, 67-74.

(24) For examples of different cyano contributions in the XPS N 1s core-level spectrum, please see: (a) Rodríguez-Pérez, L.; García, R.; Herranz, M. A.; Martín, N. Modified SWCNTs with Amphoteric Redox and Solubilizing Properties. Chem. - Eur. J. 2014, 20, 72787286. (b) Fesser, P.; Iacovita, C.; Wäckerlin, C.; Vijayaraghavan, S.; Ballav, N.; Howes, K.; Gisselbrecht, J.-P.; Crobu, M.; Boudon, C.; Stöhr, M.; Jung, T. A.; Diederich, F. Visualizing the Product of a Formal Cycloaddition of 7,7,8,8-Tetracyano-p-quinodimethane (TCNQ) to an Acetylene-Appended Porphyrin by Scanning Tunneling Microscopy on $\mathrm{Au}(111)$. Chem. - Eur. J. 2011, 17, 52465250.

(25) (a) Neri, G.; Scala, A.; Fazio, E.; Mineo, P. G.; Rescifina, A.; Piperno, A.; Grassia, G. Repurposing of oxazolone chemistry: gaining access to functionalized graphene nanosheets in a top-down approach from graphite. Chem. Sci. 2015, 6, 6961-6970. (b) Das, S. K.; KC, C. B.; Ohkubo, K.; Yamada, Y.; Fukuzumi, S.; D'Souza, F. Decorating single layer graphene oxide with electron donor and acceptor molecules for the study of photoinduced electron transfer. Chem. Commun. 2013, 49, 2013-2015. (c) Oelsner, C.; Herrero, M. A.; Ehli, C.; Prato, M.; Guldi, D. M. Charge Transfer Events in Semiconducting Single-Wall Carbon Nanotubes. J. Am. Chem. Soc. 2011, 133, 1869618706.

(26) García, D.; Rodríguez-Pérez, L.; Herranz, M. A.; Peña, D.; Guitián, E.; Bailey, S.; Al-Galiby, Q.; Noori, M.; Lambert, C. J.; Pérez, D.; Martín, N. A $\mathrm{C}_{60}$-aryne building block: synthesis of a hybrid allcarbon nanostructure. Chem. Commun. 2016, 52, 6677-6680.

(27) (a) Sarkar, S.; Bekyarova, E.; Niyogi, S.; Haddon, R. C. DielsAlder Chemistry of Graphite and Graphene: Graphene as Diene and Dienophile. J. Am. Chem. Soc. 2011, 133, 3324-3327. (b) Thomsen, C.; Reich, S. Raman Scattering in Carbon Nanotubes. Top. Appl. Phys. 2007, 108, 115-234. (c) Graupner, R. Raman spectroscopy of covalently functionalized single-wall carbon nanotubes. J. Raman Spectrosc. 2007, 38, 673-683.
(28) Gebhardt, B.; Hof, F.; Backes, C.; Müller, M.; Plocke, T.; Maultzsch, J.; Thomsen, C.; Hauke, F.; Hirsch, A. Selective Polycarboxylation of Semiconducting Single-Walled Carbon Nanotubes by Reductive Sidewall Functionalization. J. Am. Chem. Soc. 2011, 133, 19459-19473.

(29) Hof, F.; Bosch, S.; Eigler, S.; Hauke, F.; Hirsch, A. New Basic Insight into Reductive Functionalization Sequences of Single Walled Carbon Nanotubes (SWCNTs). J. Am. Chem. Soc. 2013, 135, 1838518395.

(30) (a) Maciel, I. O.; Anderson, N.; Pimenta, M. A.; Hartschuh, A.; Qian, H.; Terrones, M.; Terrones, H.; Campos-Delgado, J.; Rao, A. M.; Novotny, L.; Jorio, A. Electron and phonon renormalization near charged defects in carbon nanotubes. Nat. Mater. 2008, 7, 878-883. (b) Voggu, R.; Rout, C. S.; Franklin, A. D.; Fisher, T. S.; Rao, C. N. R. Extraordinary Sensitivity of the Electronic Structure and Properties of Single-Walled Carbon Nanotubes to Molecular Charge-Transfer. J. Phys. Chem. C 2008, 112, 13053-13056.

(31) Ragoussi, M.-E.; Malig, J.; Katsukis, G.; Butz, B.; Spiecker, E.; de la Torre, G.; Torres, T.; Guldi, D. M. Linking Photo- and Redoxactive Phthalocyanines Covalently to Graphene. Angew. Chem., Int. Ed. 2012, $51,6421-6425$.

(32) Malard, L. M.; Pimenta, M. A.; Dresselhaus, G.; Dresselhaus, M. S. Raman spectroscopy in graphene. Phys. Rep. 2009, 473, 51-87.

(33) Dyke, C. A.; Tour, J. M. Covalent Functionalization of SingleWalled Carbon Nanotubes for Materials Applications. J. Phys. Chem. A 2004, 108, 11151-11159. 